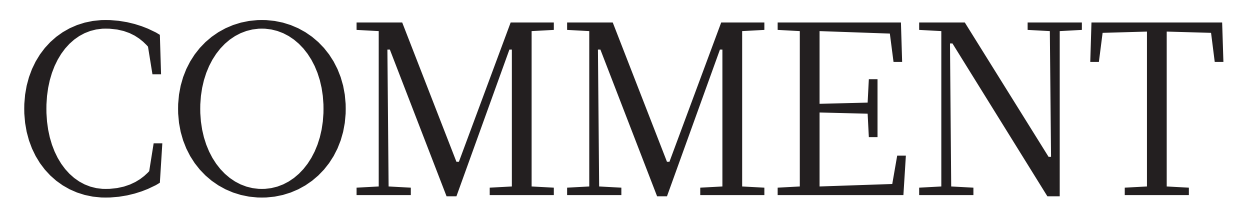

INNOVATION Twelve principles for cross-sector collaboration p.314
ECOLOGY Jane Lubchenco

enjoys a memoir of science

among the sea otters p.318
ART Wilderness photography at the improved San Francisco modern-art museum $\mathbf{p . 3 2 0}$
OBITUARY Harry Elderfield, ocean geochemist, remembered $\mathbf{p . 3 2 2}$

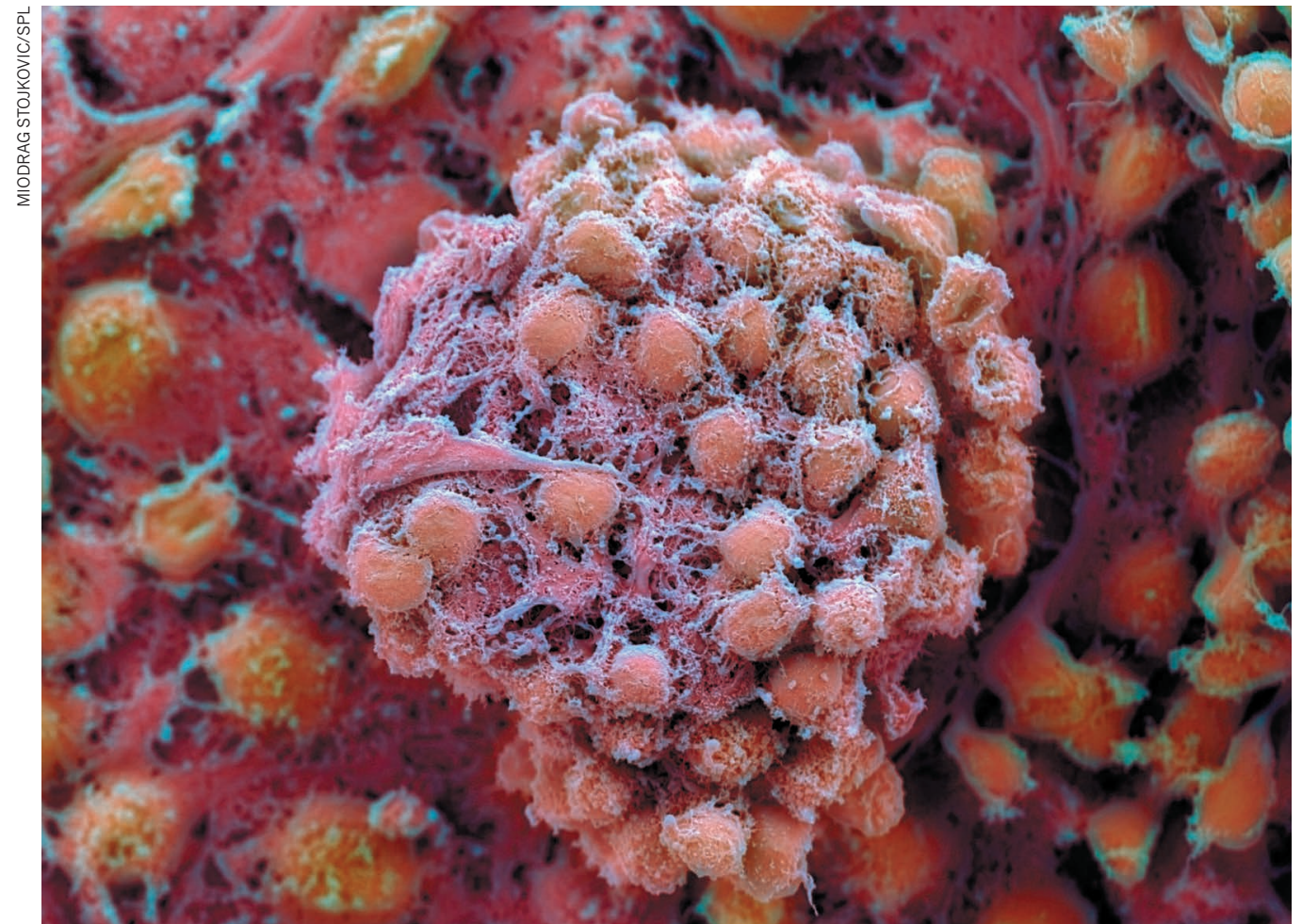

Human embryonic stem cells in culture.

\title{
Global standards for stem-cell research
}

\section{New guidelines from the International Society for Stem Cell Research offer a model for self-regulation in contentious areas, write Jonathan Kimmelman and colleagues.}

S tem-cell research offers tremendous promise for biomedicine. It also raises vexing ethical and policy challenges. It can involve the destruction, creation and modification of human embryos, and has led to the premature marketing and use of unproven therapies.

On 12 May, in response to scientific progress and evolving ethical concerns, the International Society for Stem Cell Research (ISSCR) issued updated and extended guidelines ${ }^{1}$ for work involving the manipulation of stem cells and the translation of that work into medical therapies. The ISSCR is an independent non-profit organization that was established in 2002 to provide a forum for communication and education in the emerging field of stem-cell research and regenerative medicine. The society developed guidelines for embryonic-stem-cell research ${ }^{2}$ in 2006 and for clinical translation of stem-cell research ${ }^{3}$ in 2008 . We represent the working groups that drew up the new guidelines.

The revised ISSCR guidelines provide a model of self-regulation for other potentially contentious research areas. Today's science engages many different actors: researchers, taxpayers, regulators, journals, sponsors, industries and, often, patients. Meanwhile, manuscripts, protocols, tissues and even patients routinely cross national boundaries. In this landscape, different stakeholders need to be confident that their interests will be protected when they collaborate with parties who might have differing views or goals. International guidelines are better positioned than national laws to help ensure protection.

The new ISSCR guidelines span 27 pages. Here we highlight the most dynamic areas for policy, from the introduction of 
heritable changes into the human genome to the use of sham surgical procedures in the testing of cell-based interventions.

\section{RESEARCH CHALLENGES}

Human embryos. In the decade since the ISSCR's previous guidelines were issued, embryo research has entered new arenas. Mitochondrial-replacement techniques (MRT) may soon be used to replace dysfunctional mitochondria in eggs or embryos with those obtained from healthy donors. In the United Kingdom, a pathway for bringing this approach to clinics was approved last year. And a committee convened by the National Academy of Medicine provided recommendations in February to the Food and Drug Administration that would enable clinical testing in the United States.

Mitochondrial diseases result in debilitating physical, developmental and cognitive disabilities. MRT could reduce the chances of women passing mutations associated with these diseases on to their children, but the

"A growing body of research requires the use of fresh human eggs." risks that are poorly understood.

More-contentious gene-editing techniques such as CRISPRCas9 now enable researchers to modify the nuclear DNA of human sperm and eggs (gametes) and embryos. As with MRT, there are uncertainties about the safety of these techniques. Both MRT and editing the nuclear DNA of human gametes or embryos would introduce potentially heritable alterations into the human genome. Societal consensus is lacking on whether making changes that can be inherited to the genomes of individuals is something that humankind should pursue.

Because of this, the new ISSCR guidelines assert that any attempt to modify the nuclear genome of human embryos for the purpose of human reproduction be prohibited at this time. The revised guidelines do, however, endorse continued laboratory-based research on human embryos and the derivation of stem-cell lines from them.

Just after the first ISSCR guidelines were issued in 2006, scientists reported the derivation of induced pluripotent stem (iPS) cells. These are adult cells that are reprogrammed to an embryonic-like state. Although enormously valuable, iPS cells do not obviate the need for human embryonic stem cells in research ${ }^{4}$. In fact, a better understanding of the different states of pluripotency has renewed biologists' interest in deriving embryonic stem cells with distinct properties. It has also led to a growing recognition that common animal models inadequately recapitulate many aspects of human embryonic development ${ }^{5}$.

For research involving human embryos, the revised guidelines assert the need for a specialized embryo-research-oversight (EMRO) process.

Institutions are best positioned to decide what specific mechanism to use to review embryo research. One option may be to repurpose existing embryonic-stem-cell research oversight (ESCRO) committees to take on a broader embryo-research-oversight function. Regardless of process, researchers and reviewers are urged to adhere to certain ethical principles. Among the 'points to consider' listed by the ISSCR are: whether donors of eggs or embryos have provided informed consent; the justification for the study; the number of embryos that will be used; and the quality of the study design.

The principles embodied by the revised EMRO process should be applied to the development of MRT, and to investigations of embryo-like structures, which are being increasingly used to model various stages of human development in the lab ${ }^{6}$. Such experiments warrant rigorous EMRO review to eliminate prospects that structures with the potential for integrated human organismal development are kept in vitro for anything more than the minimal periods required to address compelling scientific questions.

Human eggs. A growing body of research requires the use of fresh human eggs, whether in mitochondrial replacement, gene editing in vitro or nuclear transfer (a form of cloning for research).

Egg donation is invasive and time-consuming: it involves hormone treatment and the retrieval of eggs by needle biopsy. There are also uncertainties about its long-term effects. The practice raises several issues, including how to compensate women for the risks and discomfort but avoid economic exploitation. The new guidelines propose standards. They recommend that when women are paid, the compensation is in line with that received by volunteers in other research ${ }^{7}$.

\section{ON THE UP}

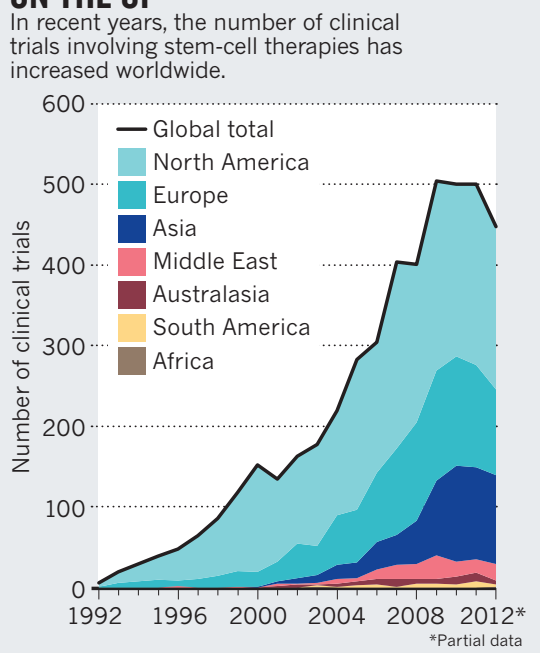

Human-animal chimaeras. Various groups are implanting human tissues into the bodies or brains of pigs, non-human primates or rodents. The resulting 'chimaeric' organisms are used to study human organ development and aspects of brain function, and to establish models of human cancer. Such transfers of human tissue raise questions about animal welfare and the limits of permissible chimaera research: it could alter animal cognition or pain perception, or lead to the formation of human gametes in the target animal.

The uncertainty over what is ethically defensible in this area was made apparent last year. The US National Institutes of Health suspended its funding of certain categories of animal-human chimaera research to first evaluate "the ethical issues that should be considered, and the relevant animal welfare concerns"

The ISSCR guidelines offer standards for researchers and reviewers that draw on welfare considerations that are broadly applicable to transgenic animals. They also recommend that certain categories of experiments be prohibited, such as the breeding of non-human animals that might harbour human gametes.

Induced pluripotent stem cells. It is unclear in many national policies whether studies that involve iPS cells should undergo a specialized stem-cell research oversight (SCRO) or an ESCRO review. The ISSCR guidelines recommend that iPS cell work be instead subject to institutional oversight of studies involving human participants, supplemented with stem-cell-relevant informed consent procedures. This would free up SCRO or ethical-review committees to focus on ethically sensitive research activities involving embryos.

\section{CLINICAL CHALLENGES}

Irreproducible results and the incomplete reporting of findings from preclinical studies are of particular concern for emerging interventions involving the transfer of living human cells. Decades of research have yielded some general insights about the behaviour of drugs in people. By contrast, the mechanisms underlying potential cellular therapies remain poorly understood for most tissues. And unlike drugs, which are metabolized and excreted from the body, stem cells and their progeny can persist, sometimes for life.

The ISSCR's 2016 guidelines articulate a detailed set of expectations regarding the design, reporting and systematic review of preclinical evidence. For instance, they advocate that the results of all preclinical studies - positive, negative and inconclusive - be reported in peer-reviewed journals and that investigators conduct a systematic 


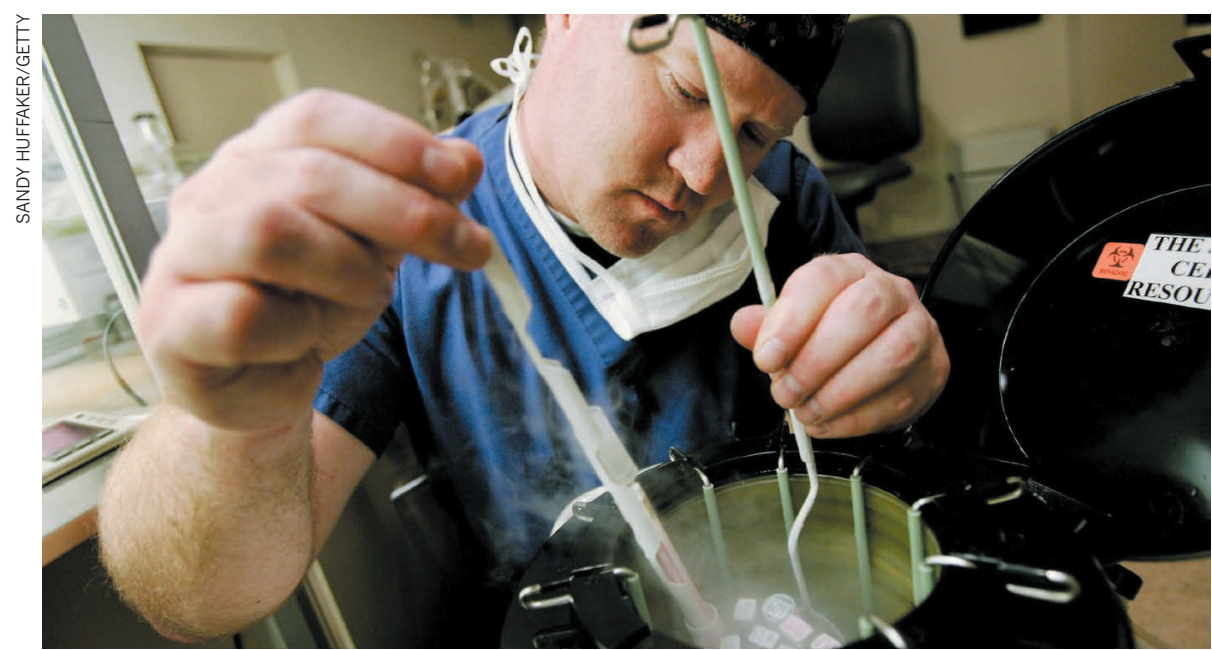

Researchers store human embryos donated for research in liquid nitrogen.

review to capture all relevant information before initiating a clinical trial (see 'On the up'). They also stipulate that trials begin only after investigators have achieved a high standard of safety and efficacy in relevant preclinical research, as determined by an independent peer-review process.

The guidelines contain strong statements about transparency in clinical research and endorse the prospective registration of all trials in public databases, regardless of phase. They also call for complete and accurate reporting of results in accordance with standards such as those provided by the CONSORT statement - an evidence-based set of recommendations for reporting randomized trials (see www.consort-statement.org).

Despite vigorous admonitions by scientists and regulators against the premature clinical translation of stem-cell therapies ${ }^{9}$, numerous providers continue to deliver illdefined cell preparations to patients outside of trials. In 2014, for instance, leaked documents from an investigation showed that a provider of an unproven cell-based intervention in Italy had no mechanism for screening cells for pathogens, and that sections of its protocol had been lifted from Wikipedia.

Such practices are worrisome. The adverse effects on people's health that may result threaten to set back more painstaking and methodical research programmes by increasing anxiety in ordinary citizens and in regulators about stem-cell-based medicines. The revised guidelines reiterate the condemnation of such practices, and consider the proper testing of new stem-cell interventions in the context of rigorous trials a matter of professional responsibility.

Increasingly, trials are being funded by patient communities, or people are paying to participate. Patient-funding may facilitate some trials that wouldn't otherwise have been pursued - such as those aimed at helping people with rare conditions. But it also allows projects to bypass the long-established mechanisms of peer review and independent oversight that encourage scientific rigour and safety. The ISSCR guidelines stipulate that studies involving paying patients are permissible only when they are subject to independent review mechanisms that can assess scientific rationale, priority and design.

The marketing of unproven interventions has happened in part because of exaggerated claims about stem-cell treatments in the media. Accordingly, the revised guidelines describe ways in which researchers can responsibly communicate with the public, emphasizing the need for balance, clarity and the avoidance of unrealistic optimism.

\section{EVER EVOLVING}

The ISSCR's 2016 guidelines are not intended to be the only or last word. They were developed by 25 scientists and ethicists from Asia, Europe, North America and Australia, with review and feedback from more than 100 individuals and organizations, including regulators, funding bodies, journal editors, patient advocates, researchers and members of the public. Some will consider aspects of the guidelines too permissive; others will find parts too restrictive. Moreover, some countries have well-articulated policies that may supersede them. The UK Human Fertilisation and Embryology Authority, for instance, has well-developed guidance on the use of MRT.

Even as we were finalizing the latest guidelines, new ethical challenges were emerging - such as questions about biosafety; whether experimentally generated, self-organizing embryonic tissues should be treated in the same way as human embryos; and whether to revisit the '14-day rule' that limits the culturing of human-embryos in vitro to two weeks ${ }^{6}$. The guidelines are intended to be revisited as science and social priorities evolve.

The many limitations of such an aspirational document notwithstanding, we believe that the ISSCR guidelines are well positioned to secure a common ethical basis for stem-cell research.

When widely embraced, international guidelines for professional conduct can be more effective than laws and regulations. The latter are confined to single jurisdictions, can be blunt regulatory instruments and change too slowly to keep pace with cutting-edge research. In highly politicized areas of science, legislated regulatory frameworks are at risk of being revised with every change in government, creating unpredictability that wastes resources and frustrates medical advances.

Guidelines are not binding, but countries, funders, journals and academic institutions can incorporate them into their policies or use them to foster a culture of compliance. Backed by evidence and sound reasoning, they can even provide evidence of professional standards to courts ${ }^{10}$. Ultimately, guidelines give voice to norms and expectations regarding preclinical evidence, trial design and independent review, providing a structured basis for adjudicating disputes.

The ISSCR guidelines continue the tradition of scientists creating professional standards for the responsible conduct of research. They speak most directly to those engaged in stem-cell research but are also relevant to regulators, journal editors, press officers, physicians, funding bodies and patients. Such a global effort to establish research standards offers a model for other contentious research arenas - from artificial intelligence to climate engineering.

Jonathan Kimmelman is associate professor in biomedical ethics, McGill University, Montreal, Canada. Insoo Hyun, Nissim Benvenisty, Timothy Caulfield, Helen E. Heslop, Charles E. Murry, Douglas Sipp, Lorenz Studer, Jeremy Sugarman and George Q. Daley. On behalf of the steering committee of the International Society for Stem Cell Research's Guidelines Task Force. e-mail: jonathan.kimmelman@mcgill.ca; george.daley@childrens.harvard.edu

1. International Society for Stem Cell Research Guidelines for Stem Cell Science and Clinical Translation (ISSCR, 2016); available at http:// www.isscr.org/guidelines2016

2. Daley, G. Q. et al. Science 315, 603-604 (2007).

3. Hyun, I. et al. Cell Stem Cell 3, 607-609 (2008).

4. Hyun, I., Hochedlinger, K., Jaenisch, R. \& Yamanaka, S. Cell Stem Cell 1, 367-368 (2007).

5. De Los Angeles, A. et al. Nature 525, 469-478 (2015).

6. Hyun, I., Wilkerson, A. \& Johnston, J. et al. Nature 533, 169-171 (2016).

7. Haimes, E. et al. Cell Stem Cell 12, 285-291 (2013)

8. US National Institutes of Health. NIH Research Involving Introduction of Human Pluripotent Cells into Non-Human Vertebrate Animal PreGastrulation Embryos (NIH, 2015).

9. Taylor, P. L. et al. Cell Stem Cell 7, 43-49 (2010). 10.Campbell, A. \& Glass, K. C. McGill Law J. 46, 473-489 (2001).

Full author affiliations and a list of co-signatories accompany this Comment online. Some authors declare competing financial interests. See go.nature.com/pcimjy for details. 